\title{
Predicting therapeutic outcome in severe ulcerative colitis by measuring in vitro steroid sensitivity of proliferating peripheral blood lymphocytes
}

\author{
S D Hearing, M Norman, C S J Probert, N Haslam, C M Dayan
}

\begin{abstract}
Background-Up to $29 \%$ of patients with severe ulcerative colitis (UC) fail to respond to steroid treatment and require surgery. Previous studies have failed to show a clear correlation between failure of steroid treatment in severe UC and measures of disease severity. The reasons for treatment failure therefore remain unknown.

Aim-To investigate the hypothesis that patients with severe UC who fail to respond to steroid treatment have steroid resistant $T$ lymphocytes.

Methods-Eighteen patients with severe UC were studied. After seven days' treatment with high dose intravenous steroids they were classified as complete responders (CR), incomplete responders (IR), or treatment failures (TF). Within 48 hours of admission blood was taken and the antiproliferative effect of dexamethasone on phytohaemagglutinin stimulated peripheral blood $\mathbf{T}$ lymphocytes was measured. Maximum dexamethasone induced inhibition of proliferation $\left(I_{\max }\right)$ was measured.

Results-In vitro $T$ lymphocyte steroid sensitivity of TF and IR patients was significantly less than that of CR patients. Both TF and 3/5 IR patients had an $I_{\max }$ of less than $60 \%$; all CR patients had an $I_{\max }$ of greater than $60 \%$. No significant correlation was seen between response to treatment and disease severity on admission. When in vitro $T$ lymphocyte steroid sensitivity was remeasured three months later, there was no difference between the groups.
\end{abstract}

Conclusions-Results suggest that T lymphocyte steroid resistance is an important

University of Bristol, Division of Medicine, Bristol Royal

Infirmary, Bristol, UK

$S$ D Hearing

M Norman

C S J Probert

N Haslam

C M Dayan

Correspondence to: Dr S D Hearing,

c/o Dr Hughes's secretary,

Department of Medicine,

Southmead Hospital,

Westbury-on-Trym, Bristol

BS10 5NB, UK.

Accepted for publication 24 February 1999

Although the aetiology of ulcerative colitis (UC) remains uncertain, ${ }^{1}$ there is a major inflammatory component to the pathological process in this disease. ${ }^{2}$ Glucocorticoids remain the mainstay of treatment for acute exacerbations. ${ }^{3}$ How- ever, up to $29 \%$ of patients with severe UC will fail to respond to steroids acutely and require surgery. ${ }^{4-6}$ In addition, some patients will require additional immunomodulatory treatment because of a poor response to steroids. ${ }^{7}$ This is a significant clinical problem in the management of these patients.

It is often assumed that responsiveness to steroid treatment in UC is simply a function of disease severity. As a result, the dose of glucocorticoid used is often chosen according to estimates of disease activity, with high dose steroid treatment being given to patients with a severe exacerbation. ${ }^{4}$ However, some mild cases of UC also fail to respond to steroid treatment. ${ }^{8}$ The reasons underlying the failure of a significant proportion of patients with UC to respond to steroids, therefore, remain uncertain.

Steroid treatment in most conditions, including UC, is given to suppress inflammation. Lymphocytes are important mediators of inflammation in many conditions including $\mathrm{UC},{ }^{9}$ and hence the response of these cells to glucocorticoids might be expected to be relevant to the outcome of steroid treatment. Peripheral blood $\mathrm{T}$ lymphocytes can be induced to proliferate in vitro using the mitogen phytohaemagglutinin (PHA). This proliferation is inhibited by glucocorticoids and the degree of inhibition of $\mathrm{T}$ lymphocyte proliferation by dexamethasone has been used to estimate steroid responsiveness. ${ }^{10}$

In recent years, there has been increasing interest in resistance to steroid treatment in conditions other than UC. In bronchial asthma, a subset of patients has been identified who, although responsive to $\beta_{2}$ agonist treatment, completely fail to respond to steroid treatment. ${ }^{11}$ Steroid resistance was found to be independent of initial lung function but correlated better with in vitro lymphocyte steroid sensitivity. ${ }^{12}{ }^{13} \mathrm{~A}$ similar correlation between in vitro lymphocyte steroid sensitivity and in vivo response to steroid treatment has been shown in rheumatoid arthritis ${ }^{14}$ and in patients receiving renal transplants. ${ }^{15}$ We therefore investigated the hypothesis that patients with severe UC who fail to respond to steroid treatment have steroid resistant $\mathrm{T}$ lymphocytes and can
Abbreviations used in this paper: 5-ASA,

5-aminosalicylic acid; CR, complete responder; CRP, C-reactive protein; GR, glucocorticoid receptor; IR, incomplete responder; PBMC, peripheral blood mononuclear cell; PHA, phytohaemagglutinin; TF, treatment failure; UC, ulcerative colitis. 
be identified prospectively by measuring in vitro $\mathrm{T}$ lymphocyte steroid sensitivity.

\section{Methods}

PATIENT CHARACTERISTICS

All patients with severe UC, according to Truelove and Witts criteria, ${ }^{16}$ admitted over one year to a single centre (Bristol Royal Infirmary) were recruited. Truelove and Witts criteria are: six or more bloody stools per day, with one or more of: tachycardia (pulse greater than 90 beats per minute), pyrexia (temperature above $37.8^{\circ} \mathrm{C}$ ), erythrocyte sedimentation rate (ESR) greater than $30 \mathrm{~mm} / \mathrm{h}$, plasma viscosity greater than $1.85 \mathrm{mPa}$ (normal range 1.55-1.75), or anaemia (haemoglobin less than $10.5 \mathrm{~g} / \mathrm{dl}$ ).

Exclusion criteria were: age less than 18 years; known Crohn's disease; or presence on admission of an indication for immediate surgery. Previous or current steroid treatment or 5-aminosalicylic acid (5-ASA) treatment were not exclusion criteria. Written informed consent was obtained from each patient and the Local Research Ethics Committee gave permission for this study.

MONITORING

Demographic data for each patient were recorded. Date of diagnosis, current treatment, and disease extent (using established endoscopic or radiological criteria) $)^{17}$ were also recorded. A plain abdominal radiograph was taken on admission, a rigid sigmoidoscopy was performed, and stool samples collected for microscopy, culture, and measurement of Clostridium difficile toxin. All patients were transferred to the care of a gastroenterologist and treatment instituted according to a standard protocol (see below). Temperature, and pulse and blood pressure were recorded every six hours and a stool chart recording frequency, consistency, amount, and visible blood was kept. Full blood count, plasma viscosity, urea, electrolytes, serum albumin, and $\mathrm{C}$ reactive protein (CRP) measurements were made on admission and repeated at least every two days subsequently. A $20 \mathrm{ml}$ heparinised blood sample was taken within 48 hours of admission, before or after commencing steroid treatment. This sample was obtained between 900 and $1000 \mathrm{am}$ and was used for cortisol measurement, and peripheral blood mononuclear cell (PBMC) separation with measurement of in vitro $\mathrm{T}$ lymphocyte steroid sensitivity.

MANAGEMENT

A standard treatment protocol was used to ensure that differences in clinical response were not due to differences in the treatment given. Steroid treatment was hydrocortisone $100 \mathrm{mg}$ four times daily, given intravenously to eliminate malabsorption of medication as a possible cause for treatment failure. Additional treatment consisted of intravenous fluids to correct fluid and electrolyte deficiency, blood transfusion to keep haemoglobin above $10 \mathrm{~g} / \mathrm{dl}$ (in all cases after samples for lymphocyte separation had been obtained), unfractionated heparin subcutaneously $5000 \mathrm{U}$ twice daily, continued oral 5-ASA treatment if already being taken, bed rest, food and oral fluids as requested by patient, nutritional assessment, and supplementation with Fortisip if body mass index was below 20 or serum albumin less than $32 \mathrm{~g} / 1$.

OUTCOME

After seven days treatment patient outcome was classified in terms of response according to the following standard criteria ${ }^{6}$ : complete response (CR) - three or less stools per day, with no visible blood; incomplete response (IR) - four or more stools per day or visible blood but no indication for colectomy (see below); or treatment failure (TF)-indication for colectomy present (complete failure to respond or deterioration within first seven days, perforation, increasing colonic dilatation, or massive haemorrhage).

Patients were recalled after three months and in vitro $\mathrm{T}$ lymphocyte steroid sensitivity remeasured. In addition all disease activity indices were reassessed.

\section{MEASUREMENT OF IN VITRO T LYMPHOCYTE} STEROID SENSITIVITY

The sensitivity to glucocorticoids of peripheral blood $\mathrm{T}$ lymphocytes from patients was assessed in a blinded fashion (the laboratory operator was unaware of the clinical status of the patient). PBMC were isolated from $20 \mathrm{ml}$ of heparinised blood by buoyant density centrifugation. The sensitivity of proliferation to suppression by dexamethasone was then assessed as previously described. ${ }^{12}$ PBMC were incubated at a concentration of $4 \times 10^{5}$ cells/well in RPMI-1640 medium (Gibco, Life Technologies Ltd, Paisley, UK) containing $10 \%$ foetal calf serum (Gibco) in 96 well round bottomed microtitre plates (Nunc, Life Technologies Ltd, Paisley, UK). The T lymphocyte mitogen PHA (Sigma Aldrich Company Ltd, Poole, UK) was added to the cultured cells at a final concentration of $5 \mu \mathrm{g} / \mathrm{ml}$, along with dexamethasone (Sigma) at increasing concentrations in the range $10^{-11}-10^{-6} \mathrm{~mol} / 1$. Cultures were then incubated at $37^{\circ} \mathrm{C}$ in a humidified atmosphere with $5 \% \mathrm{CO}_{2}$ for 48 hours. Cell proliferation was measured by uptake of tritiated thymidine. Sterile [methyl- $\left.{ }^{3} \mathrm{H}\right]-$ thymidine (Amersham International plc, Little Chalfont, UK; $0.0185 \mathrm{MBq} /$ well in a volume of $10 \mu \mathrm{l})$ was added to cell culture wells for the last six hours of incubation. Cells were then harvested onto a glass fibre filter paper (Wallac Oy, Turku, Finland) using cell harvester apparatus (Tomtec, Orange, Connecticut, USA) and the incorporated radiolabel was counted using a Micro $\beta$ emission scintillation counter (Wallac). Results were calculated as mean counts per minute of triplicate cultures.

\section{STATISTICAL ANALYSIS}

Inhibition of thymidine uptake for each concentration of dexamethasone was compared with uninhibited control values (PHA stimulated $\mathrm{T}$ lymphocytes in the absence of dexamethasone) and expressed as percentage suppression. For each subject, sigmoid doseresponse curves for inhibition of proliferation by dexamethasone were fitted using non-linear 
Table 1 Individual patient characteristics

\begin{tabular}{|c|c|c|c|c|c|}
\hline Patient & $\begin{array}{l}\text { Duration of } \\
\text { UC }\end{array}$ & $\begin{array}{l}\text { Preadmission } \\
\text { 5-ASA? }\end{array}$ & Preadmission steroids? & $\begin{array}{l}\text { Duration of steroids before } \\
\text { blood taken } *\end{array}$ & $\begin{array}{l}\text { Response to } \\
\text { treatment }\end{array}$ \\
\hline 1 & New & No & No & $<24$ hours & $\mathrm{CR}$ \\
\hline 2 & 9 years & Yes & No & $<24$ hours & IR \\
\hline 3 & 5 months & Yes & No & $<24$ hours & $\mathrm{TF}$ \\
\hline 4 & 6 years & No & Prednisolone $5 \mathrm{mg}$ daily & $<48$ hours & IR \\
\hline 5 & New & No & No & Before steroids & $\mathrm{TF}$ \\
\hline 6 & New & No & No & $<24$ hours & $\mathrm{CR}$ \\
\hline 7 & 1 month & No & Colifoam enema only & $<24$ hours & $\mathrm{CR}$ \\
\hline 8 & New & No & No & $<24$ hours & $\mathrm{CR}$ \\
\hline 9 & 3 years & No & No & Before steroids & IR \\
\hline 10 & 17 years & Yes & Colifoam enema only & $<48$ hours & $\mathrm{CR}$ \\
\hline 11 & 19 years & Yes & Prednisolone $10 \mathrm{mg}$ daily & $<24$ hours & $\mathrm{CR}$ \\
\hline 12 & 7 months & No & No & $<48$ hours & IR \\
\hline 13 & 10 years & No & Prednisolone $30 \mathrm{mg}$ daily & $<24$ hours & $\mathrm{CR}$ \\
\hline 14 & 9 months & Yes & No & $<24$ hours & $\mathrm{CR}$ \\
\hline 15 & New & No & No & $<48$ hours & $\mathrm{CR}$ \\
\hline 16 & New & No & No & Before steroids & IR \\
\hline 17 & 9 years & No & No & $<48$ hours & $\mathrm{CR}$ \\
\hline 18 & 4 years & Yes & No & Before steroids & $\mathrm{CR}$ \\
\hline
\end{tabular}

* Refers to the duration of high dose intravenous hydrocortisone given before blood was taken for PBMC separation and measurement of serum cortisol.

5-ASA, 5-aminosalicylic acid; UC, ulcerative colitis; CR, complete responder; IR, incomplete responder; TF, treatment failure.

regression. The exact value of maximum inhibition of proliferation $\left(I_{\max }\right)$ cannot be measured as it is the inhibition seen at an infinitely high level of dexamethasone. In our study the percentage inhibition observed at the maximum dexamethasone concentration applied $\left(10^{-6} \mathrm{~mol} / \mathrm{l}\right)$ was therefore used as the measure of $I_{\max }$ for all subjects. In practice, there was good agreement between this value and the estimated asymptotic value for maximal inhibition obtained from the doseresponse curves (results not shown).

The Kruskal-Wallis test was used to compare the steroid sensitivity in all three groups and test whether there was an overall difference in sensitivity among the three response groups. Following power calculations, a decision was made prior to commencing the study to combine the clinical response groups, $\mathrm{TF}$ and IR, and compare this combined group with the CR group for all further statistical analysis. Comparison of the degree of inhibition of in vitro $\mathrm{T}$ lymphocyte proliferation at each concentration of dexamethasone was made using the Mann-Whitney U test for each clinical response patient group. Truelove and Witts

Table 2 Admission data

\begin{tabular}{|c|c|c|c|c|}
\hline & \multicolumn{2}{|c|}{ Complete responders } & \multicolumn{2}{|c|}{$\begin{array}{l}\text { Incomplete responders and } \\
\text { treatment failures }\end{array}$} \\
\hline Number of patients & 11 & & 7 & \\
\hline First presentation (\%) & 36 & & 28 & \\
\hline Age (y) & 35 & $(27-67)$ & 50 & $(36-55)$ \\
\hline Male sex $(\%)$ & 72 & & 71 & \\
\hline Stools/day & 8 & $(7-10)$ & 10 & $(6-10)$ \\
\hline Bloody stools/day & 7 & $(6-10)$ & 6 & $(6-7)$ \\
\hline Temperature $\left({ }^{\circ} \mathrm{C}\right)$ & 37.2 & $(37.0-38.0)$ & 37.5 & $(37.5-38.0)$ \\
\hline Pulse rate (beats $/ \mathrm{min}$ ) & 96 & $(84-104)$ & 80 & $(80-100)$ \\
\hline Haemoglobin (g/dl) & 12.1 & $(10.2-12.8)$ & 12.2 & $(9.3-14.3)$ \\
\hline Plasma viscosity $(\mathrm{mPa})$ & 1.75 & $(1.64-1.88)$ & 1.88 & $(1.75-2.17)$ \\
\hline White cell count $\left(10^{9} / 1\right)$ & 9.5 & $(8.1-16.1)$ & 11.5 & $(8.8-12.9)$ \\
\hline Lymphocyte count $\left(10^{9} / 1\right)$ & 2.0 & $(0.9-3.3)$ & 1.2 & $(0.7-1.9)$ \\
\hline Platelet count $\left(10^{9} / 1\right)$ & 378 & $(333-396)$ & 478 & $(320-643)$ \\
\hline C-reactive protein $(\mathrm{mg} / \mathrm{l})$ & 38 & $(13-66)$ & 57 & $(18-68)$ \\
\hline Albumin $(\mathrm{g} / \mathrm{l})$ & 40 & $(35-42)$ & 34 & $(32-42)$ \\
\hline Cortisol (nmol/1) & 1743 & $(1101-1960)$ & 1062 & $(675-1854)$ \\
\hline Blood transfusion (units) & 0 & $(0-0)$ & 0 & $(0-2)$ \\
\hline Sigmoidoscopic appearance (grade) & 3 & $(3-4)$ & 4 & $(3-4)$ \\
\hline Pancolitis or extensive disease $(\%)$ & 55 & & 71 & \\
\hline Left sided or distal disease $(\%)$ & 45 & & 29 & \\
\hline
\end{tabular}

Values are medians (interquartile range). No significant differences between the groups according to any of the parameters measured were seen. For male sex, first presentation and extent of disease $\%$ of population is given and no significant association with clinical outcome was seen. criteria for disease severity, haematological and biochemical measurements, and patient age were also compared with response to treatment using the Mann-Whitney U test. Fisher's exact test was used to test the association between $I_{\max }$ and clinical response of severe UC to treatment. Predictive values and confidence intervals were calculated for $I_{\max }$. Fisher's exact test was also used to test the association between the non-continuously variable data (sex, first presentation, and extent of disease) and clinical response of severe UC to treatment.

\section{Results}

CLINICAL RESPONSE

Eighteen consecutive patients were recruited to the study. After seven days treatment, 11 patients achieved a complete response (CR), five an incomplete response (IR), and two patients were treatment failures $(\mathrm{TF})$, requiring emergency colectomy after complete failure to respond to seven days steroid treatment. These results (61\% CR at seven days) are in keeping with previously documented outcomes for patients with severe UC. ${ }^{4-6}$

After three months, 17 patients were available for follow up; one patient had returned to the USA and was lost to follow up. Nine of the 11 patients achieving a CR remained well on oral prednisolone with or without a 5-ASA drug; the remaining two patients in this group underwent colectomy, one because of frequent steroid responsive exacerbations, and the other because of relapse when steroid treatment was reduced. Of the five patients in the IR group, three underwent colectomy (one after four weeks, one after five months, and one after 13 months); one currently requires azathioprine, oral prednisolone (currently $30 \mathrm{mg}$ once daily), and a 5-ASA drug; and one was the patient who returned to the USA. Both patients in the TF group who had undergone emergency colectomy remained well.

\section{PATIENT CHARACTERISTICS AND CLINICAL} OUTCOME

Tables 1 and 2 summarise the characteristics of patients recruited to the study. UC was newly diagnosed in six cases. Of the 12 patients 


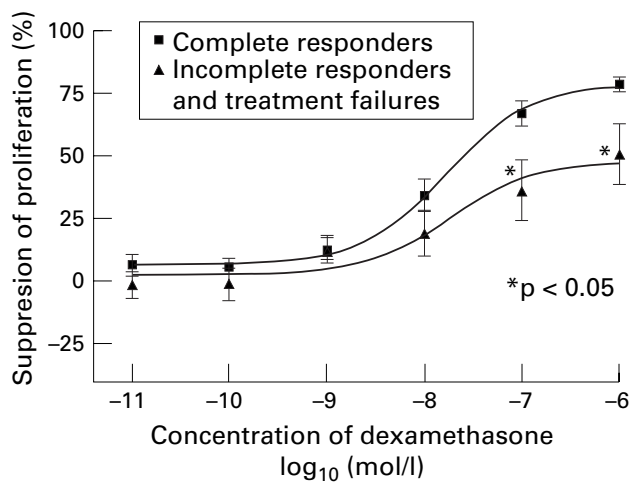

Figure 1 Inhibition of phytohaemagglutinin stimulated $T$ lymphocyte proliferation by dexamethasone for the different clinical response groups; error bars represent SEM.

Thymidine counts (mean (SD)): complete responders 54611 (28 620) cpm; patients not achieving complete response 54046 (21 644) cpm (incomplete responders 49385 (21 643) cpm, treatment failures 65696 (3562) cpm).

known to have UC, six were receiving 5-ASA treatment and three oral glucocorticoids prior to admission. All blood samples were taken between 900 and $1000 \mathrm{am}$, in all cases within 48 hours of admission. The majority of patients had been commenced on steroid treatment at the time of testing $(14 / 18)$.

The Kruskal-Wallis test was performed, comparing steroid sensitivity in all three groups. The results showed that there is an overall difference in sensitivity among the three clinical response groups $\left(\chi^{2}=6.77, \mathrm{df}=2\right.$, $\mathrm{p}=0.033)$. All subsequent statistical analysis was undertaken combining the clinical response groups TF and IR. Thus indicators of disease activity on admission were compared between patients achieving CR and patients not achieving this (IR and TF). Trends were observed in some parameters including stool frequency, plasma viscosity, white cell count, platelet count, CRP, low serum albumin, and disease extent (table 2), but none of these reached statistical significance. Serum cortisol levels were higher in patients achieving CR, although this difference did not reach statistical significance. Serum levels of cortisol in blood samples taken from patients receiving intravenous hydrocortisone were up to $2000 \mathrm{nmol} / \mathrm{l}$. Hydrocortisone is identical to cortisol and thus these cortisol levels represent endogenous cortisol and pharmacological hydrocortisone. The difference in serum cortisol levels between the two clinical response groups, could be accounted for by the fact that 3/7 IR/TF patients and only one CR patient had blood for PBMC separation and cortisol measurement taken before treatment with high dose intravenous steroids was commenced (table 1).

IN VITRO T LYMPHOCYTE STEROID SENSITIVITY On admission

In vitro $\mathrm{T}$ lymphocyte steroid sensitivity of $\mathrm{TF}$ and IR patients was significantly less than CR patients at dexamethasone concentrations $10^{-7}$ $\mathrm{mol} / \mathrm{l}(\mathrm{p}=0.01)$ and $10^{-6} \mathrm{~mol} / \mathrm{l}(\mathrm{p}=0.03)$ (fig 1$)$. In addition, both $\mathrm{TF}$ and $3 / 5$ IR patients had an $\mathrm{I}_{\max }$ of less than $60 \%$. In contrast all $11 \mathrm{CR}$ patients had an $I_{\max }$ greater than $60 \%$ (Fisher's exact test, $p=0.0025$ ) (fig 2 ). Positive predictive

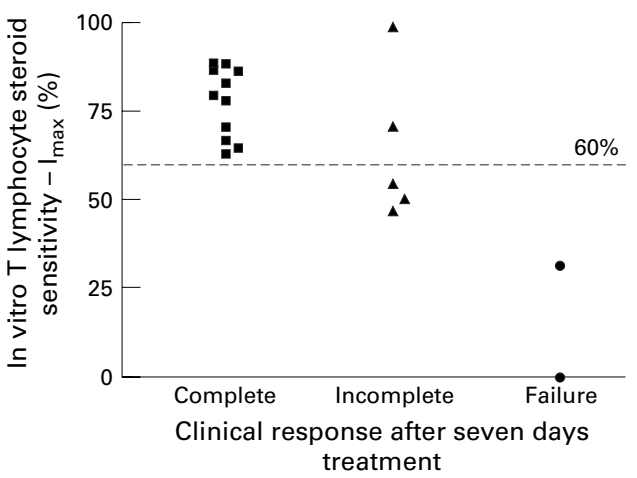

Figure 2 In vitro T lymphocyte steroid sensitivity on admission compared with clinical outcome in severe ulcerative colitis.

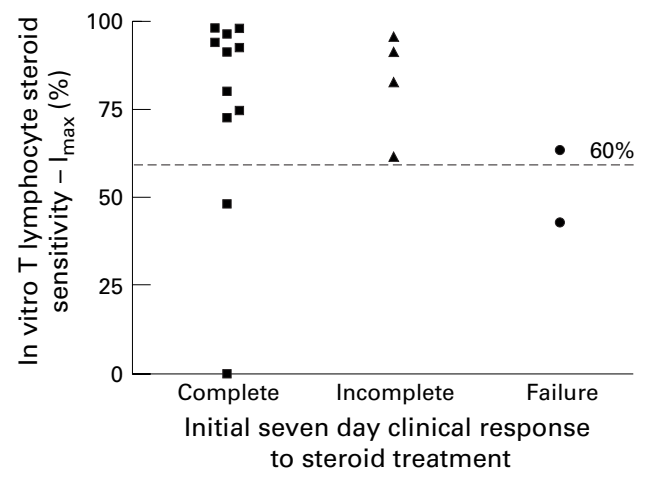

Figure 3 In vitro T lymphocyte steroid sensitivity at three months' follow up compared with initial clinical outcome in severe ulcerative colitis.

value ( $I_{\max }$ greater than $60 \%$ ) for complete response was $84 \%$ (95\% confidence intervals (CI): $64 \%$ to $97 \%$ ) and negative predictive value ( $\mathrm{I}_{\max }$ less than $\left.60 \%\right)$ was $100 \%(95 \% \mathrm{CI}$ : $55 \%$ to $100 \%$ ).

Three month follow up

When in vitro $\mathrm{T}$ lymphocyte steroid sensitivity was remeasured there was no difference between the groups (fig 3).

\section{Discussion}

The reasons underlying the high failure rate of steroid treatment in severe UC have previously been unclear. We have shown an association between in vitro measurement of T lymphocyte steroid sensitivity and clinical response to steroids in severe UC. The effect was large enough to be significant even in a small study. In contrast, there was no significant difference in admission disease severity indices between complete responders and patients not achieving complete response, although trends in some parameters were observed. The number of patients in this study is relatively small and because of this statistical non-significance in admission disease severity indices does not completely exclude differences that may have contributed to clinical response. However previous studies of severe UC have also shown little difference in admission data between responders and non-responders in keeping with our results. ${ }^{56}$ Care was taken in the 

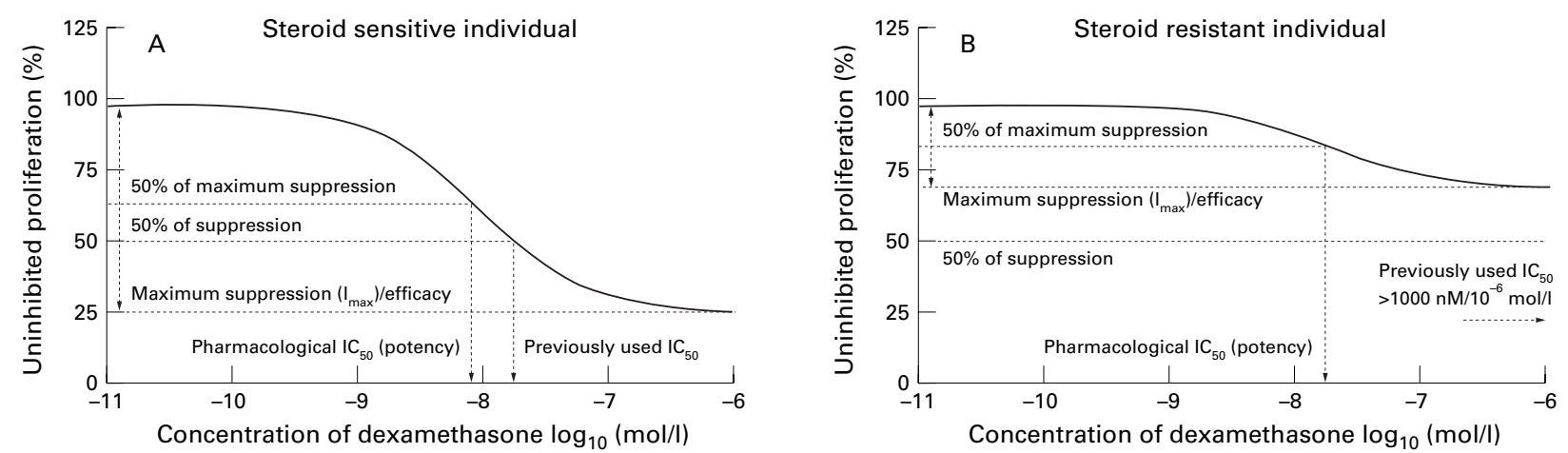

Figure 4 Definition of $I_{\text {ax }}$ and $I C_{50}$ in steroid sensitive and resistant subjects compared with previously used method of calculating IC $C_{50}$. In steroid sensitive individuals $I C_{50}$ is similar using both methods; however, when the individual is steroid resistant there is a large difference between the two methods.

present study to ensure that management of all patients was according to a standard protocol and that high dose intravenous steroids were used to eliminate medication malabsorption as a possible cause of treatment failure. ${ }^{18}$ Our data therefore raise the intriguing possibility that $\mathrm{T}$ lymphocyte sensitivity to steroids may be as, if not more, important than disease severity in determining the response to steroid treatment in severe UC.

Efforts were made in this study to minimise variation in the measure of steroid sensitivity. The lymphocyte proliferation assay used to measure in vitro steroid sensitivity had low intra-assay and interassay variation (less than $4 \%$ ) (Hearing et al, in preparation). In addition, steroid sensitivity was measured at a standard time of the day (900 to $1000 \mathrm{am}$ ) to minimise any possible circadian effects. This resulted in 14 of the 18 patients being studied after the commencement of steroid treatment (which could not be delayed). Despite this, an association between $\mathrm{T}$ lymphocyte steroid sensitivity and clinical outcome of severe UC was still shown. Whether steroid treatment causes significant changes in steroid sensitivity has not been formally studied. However, no correlation was seen between steroid sensitivity and cortisol level, either at the time of severe illness $(p=0.61)$ or at three months $(p=0.58)$. In addition, both sensitive and resistant values were obtained in samples taken both before and after commencement of parenteral glucocorticoid treatment. Hence, it seems unlikely that glucocorticoid treatment significantly affects in vitro $\mathrm{T}$ lymphocyte sensitivity.

Previous studies relating in vitro $\mathrm{T}$ lymphocyte steroid sensitivity to clinical response in other diseases have expressed steroid sensitivity as $\mathrm{IC}_{50}{ }^{101214} 15$ The definition of $\mathrm{IC}_{50}$ used in these studies was the concentration of dexamethasone producing $50 \%$ inhibition of proliferation, a parameter which incorporates both efficacy and potency. For comparison with previous studies, ${ }^{10} 1214{ }^{15}$ recalculation of our results using this definition of $\mathrm{IC}_{50}$ revealed a significant association between in vitro measurement of T lymphocyte steroid sensitivity and clinical response to steroids (Fisher's exact test, $\mathrm{p}<0.0001)$. However, in pharmacological terms, IC $_{50}$ (potency) should more properly be defined as the concentration of an agent producing $50 \%$ of the maximal inhibi- tory effect (fig 4). If $\mathrm{IC}_{50}$ is calculated from our results using this latter definition, no correlation is seen. Since $\mathrm{IC}_{50}$ calculated in this way represents potency, this simply implies that it is efficacy and not potency that is important in determining outcome. This is not surprising, since at the circulating steroid levels achieved with high dose intravenous hydrocortisone in this study glucocorticoid receptors (GRs) are likely to be more than $95 \%$ occupied. We therefore feel justified in expressing steroid sensitivity in terms of a single variable $\left(\mathrm{I}_{\max }\right)$ which equates very closely to true pharmacological efficacy.

Glucocorticoids exert their effects on cells via binding to GR in the cytoplasm. Once the GR-glucocorticoid complex is formed, it translocates to the nucleus and binds either to DNA, which typically results in gene transactivation, ${ }^{19}$ or to transcription factors such as activator protein 1 (AP1), ${ }^{20}$ typically resulting in transrepression. ${ }^{21}$ Primary steroid resistance due to inherited abnormalities of the GR has been described, ${ }^{22}$ but very few cases have been reported worldwide and none of our patients had the grossly elevated cortisol levels characteristic of this condition, when followed up (data not shown). Steroid resistance not due to gross receptor abnormalities has now been recognised as a factor influencing response to treatment in several inflammatory and immunological conditions. ${ }^{12-15}$ The range of steroid responsiveness of $\mathrm{T}$ lymphocytes found in our work is consistent with that found in these previous studies of steroid resistance. The mechanism for steroid resistance remains uncertain but exposure to cytokines in vitro has been shown to reduce peripheral blood mononuclear cell GR affinity, ${ }^{23}$ and abnormalities of GR-AP1 binding have been shown in steroid resistant asthma, suggesting a postreceptor mechanism. $^{24}$

When in vitro T lymphocyte steroid sensitivity was remeasured three months after the acute episode, steroid sensitivity of some patients had changed and the correlation with response to treatment was no longer present. This indicates that it is the level of steroid sensitivity at the time of initiating treatment which is relevant to the outcome of treatment, as might be expected. These data also suggest that $\mathrm{T}$ lymphocyte steroid sensitivity is not a fixed characteristic for some individuals. The factors 
responsible for modulating steroid sensitivity remain uncertain. As already discussed it seems that glucocorticoid levels make little difference to in vitro $\mathrm{T}$ lymphocyte steroid sensitivity and prevailing glucocorticoid concentrations are probably also insufficient to explain variations in individual steroid sensitivity. It also seems unlikely that the variation seen in individual steroid sensitivity before and after treatment simply represents a surrogate marker of disease activity as three months after the acute episode the steroid resistance had actually increased in some individuals but had fallen in others (fig 3). In particular, two patients who had initially presented with steroid sensitive lymphocytes and clinically steroid responsive disease, had steroid resistant lymphocytes when remeasured after three months, despite being in remission at that time. In addition, while steroid sensitivity in the IR patient undergoing colectomy prior to repeat assessment at three months increased, of the two patients in the TF group, steroid sensitivity increased in one patient, but the other remained steroid resistant following colectomy. An alternative explanation for this lack of correlation between steroid sensitivity and change in disease activity might be that the severity of the inflammation is not fully reflected by the traditional markers of disease activity examined in this study. However, even if this were true, this would not explain our data. We have since conducted a study of healthy individuals and found a very large range of steroid sensitivity, encompassing the level of steroid resistance seen in steroid resistant TF/IR patients (Hearing et al, in preparation). We currently believe that the variation in steroid sensitivity seen before and after treatment is a combination of individuals' baseline steroid sensitivity and a variable effect of disease.

Our results suggest that increased understanding of the factors which determine steroid sensitivity may make it possible to improve the effectiveness of steroid treatment. The maximum concentration of dexamethasone used in the in vitro lymphocyte proliferation assay was $10^{-6} \mathrm{~mol} / \mathrm{l}$ and we showed that this concentration of dexamethasone failed to inhibit the lymphocytes of steroid resistant subjects significantly. Dexamethasone $10^{-6} \mathrm{~mol} / 1$ is approximately tenfold greater in potency of glucocorticoid activity than the serum levels of hydrocortisone achieved with the treatment protocol used $(2000 \mathrm{nmol} / \mathrm{l})$. The fact that lymphocytes were not inhibited by high concentration of dexamethasone suggests that simply increasing the dose of therapeutic steroid used in severe UC is unlikely to improve the clinical response in steroid resistant subjects. Measures to increase steroid sensitivity in these steroid resistant subjects are probably required to improve the effectiveness of steroid treatment, and thus reduce the number of treatment failures in severe UC.

The findings of this study suggest that measurement of in vitro $\mathrm{T}$ lymphocyte steroid sensitivity may prove a more reliable predictor of failure to respond to treatment than any previously studied parameter. Current practice is to offer surgery to patients failing to respond to steroids after five to seven days. ${ }^{6}$ If patients have an $I_{\max }$ less than $60 \%$, our data would suggest that a complete response to medical treatment is unlikely and so early intervention with alternative immunomodulatory treatment or surgery would be indicated. The negative predictive value was $100 \%$ although the confidence intervals were wide, reflecting the small number of patients in this study. There is clearly a pressing need for our data to be confirmed in larger studies, and in particular for more treatment failure patients to be studied, as the predictive value of the measurement of lymphocyte steroid sensitivity would be of greatest clinical value in these patients. The results of in vitro $\mathrm{T}$ lymphocyte steroid sensitivity could be known within 48 hours of starting treatment and could guide decisions regarding the timing of surgery or alternative medical treatments. Knowledge of steroid resistance could prevent protracted and ultimately ineffective steroid treatment with the attendant risks of deterioration in the physical condition of the patient, increased friability of the inflamed colon, and prolonged exposure to steroids. Treatment with additional immunomodulatory therapy, such as cyclosporin, could be instituted several days earlier when it may be more effective, or surgery might be undertaken earlier, reducing the risks to the patient.

SDH was the recipient of a research training fellowship from The Digestive Disorders Foundation and The Ileostomy and Internal Pouch Support Group, and a grant from The Special Trustees for the United Bristol Hospitals. We thank Caroline Smythe for assistance with specimen collection, Professor Peter Barnes, Chris Corrigan, and Ian Adcock for technical assistance, Ken Heaton and Professor Stafford L Lightman for Swinburn, and Chris Foy for assistance with statistical analysis.

1 Shanahan F. Pathogenesis of ulcerative colitis. Lancet 1993;342:407-11.

2 Elson CO. The basis of current and future therapy for inflammatory bowel disease. Am f Med 1996;100:656-62. inflammatory bowel disease. Am f Med 1996;100:656-62. disease. Aliment Pharmacol Ther 1993;7:567-79.

4 Truelove SC, Willoughby CP, Lee EG, et al. Further experience in the treatment of severe attacks of ulcerative colitis. Lancet 1978;ii:1086-8.

5 Chakravarty BJ. Predictors and the rate of medical treatment failure in ulcerative colitis. Am $\mathcal{F}$ Gastroenterol 1993;88:852-5.

6 Travis SPL, Farrant JM, Nolan DJ, et al. Predicting outcome in severe ulcerative colitis. Gut 1996;38:905-10.

7 Lichtiger S, Present DH, Kornbluth A, et al. Cyclosporine in severe ulcerative colitis refractory to steroid therapy. $\mathrm{N} \mathrm{Engl}$ 7 Med 1994;330:1841-5.

8 Griffin MG, Miner PB. Review article: refractory distal colitis-explanations and options. Aliment Pharmacol Ther 1996;10:39-48.

9 Schreiber S, MacDermott RP, Raedler A, et al. Increased activation of isolated intestinal lamina propria mononuclear cells in inflammatory bowel disease. Gastroenterology 1991;101:1020-30.

10 Walker KB, Potter JM, House AK. Interleukin 2 synthesis in the presence of steroids: a model of steroid resistance. Clin Exp Immunol 1987;68:162-7.

11 Carmichael J, Paterson IC, Diaz P, et al. Corticosteroid resistance in chronic asthma. BMF 1981;282:1419-22.

12 Corrigan CJ, Brown PH, Barnes NC, et al. Glucocorticoid resistance in chronic asthma: glucocorticoid pharmacokinetics, glucocorticoid receptor characteristics, and inhibition of peripheral blood $\mathrm{T}$ cell proliferation by glucocorticoids in vitro. Am Rev Respir Dis 1991;144:1016-25.

13 Alvarez J, Surs W, Leung DYM, et al. Steroid-resistant asthma: immunological and pharmacological features. $\mathcal{F}$ Allergy Clin Immunol 1992;89:714-21.

14 Kirkham BW, Corkill MM, Davison SC, et al. Response to glucocorticoid treatment in rheumatoid arthritis: in vitro glucocorticoid treatment in rheumatoid arthritis: in vitro
Rheumatol 1991;18:821-5.

15 Langhoff E, Ladefoged J, Jakobsen BK, et al. Recipient lymphocyte sensitivity to methylprednisolone affects cadaver phocyte sensitivity to methylprednisolone
kidney graft survival. Lancet 1986; $1296-7$.

16 Truelove SC, Witts LJ. Cortisone in ulcerative colitis. Final report on a therapeutic trial. BMF 1955;2:1041-8. 
17 Forbes A. Frequency and significance of proximal extension of distal ulcerative colitis. Eur 7 Gastroenterol Hepatol 1996 8:543-4.

18 Frey BM, Frey FJ. Clinical pharmacokinetics of prednisone and prednisolone. Clin Pharmacokinet 1990;19:126-46.

19 Beato M. Gene regulation by steroid hormones. Cell 1989;56:335-44.

20 Schüle R, Rangarajan P, Kliewer S, et al. Functional antagonism between oncoprotein c-jun and the glucocorticoid receptor. Cell 1990;62:1217-26.

21 Paliogianni F, Raptis A, Ahuja SS, et al. Negative transcriptional regulation of human interleukin 2 (IL-2) gene by glucocorticoids through interference with nuclear tran- scription factors AP-1 and NF-AT. $\mathcal{f}$ Clin Invest 1993;91: 1481-9.

22 Iida S, Gomi M, Moriwaki K, et al. Primary cortisol resistance accompanied by a reduction in glucocorticoid receptors in two members of the same family. $\mathcal{F}$ Clin Endocrinol Metab 1985;60:967-71.

23 Kam JC, Szefler SJ, Surs W, et al. Combination IL-2 and IL-4 reduces glucocorticoid receptor-binding affinity and T cell response to glucocorticoids. F Immunol 1993;151: 3460-6.

24 Adcock IM, Lane SJ, Brown CR, et al. Abnormal glucocorticoid receptor-activator protein 1 interaction in steroidresistant asthma. $\mathcal{F}$ Exp Med 1995;182:1951-8. 